\title{
Reliability Analysis of the Energy Efficient Cluster Technique for WSN for Mobile Sink
}

\author{
Shiv Prasad Kori \\ Sr. Lecturer \\ Dept.of Electronics \& Tele. \\ JIJA Mata GovtPolytechnicCollege \\ Burhanpur, India
}

\author{
R. K .Baghel, Ph.D \\ Electronics and Communication \\ Department MANIT, Bhopal \\ India
}

\begin{abstract}
Presently wireless sensor network (WSN) is very popular in the many of industrial area where the cabling from sensor to sink is difficult. Because of growing use of WSN, it is a emerging topic in the research area. There are various limitation of WSN with high number of node and large area of network. The energy consumption is one of the important aspects of WSN due to limited access of sensor node and limited battery power. There are various research performed to reduce the energy loss in WSN by optimizing the physical layer parameter such that the modulation technique, operating frequency and antenna design. The energy loss also minimize by optimization of communication protocol. There is tradeoff between the reduction of energy consumption using protocol optimization and reliability of communication. The energy loss can be minimizing by reducing the communication overhead but it may lead to information loss during the transient behavior of WSN. The problem of reliability becomes more critical when the sink has mobility. This paper will show the implementation of simulation setup for mobile sink based WSN and performance evaluation of WSN for communication overhead and reliability. Two protocol BBM and cluster based protocol are comparing for performance analysis.
\end{abstract}

\section{Keyword}

WSN, cluster, BBM, reliability, communication overhead etc.

\section{INTRODUCTION}

The wireless network has become a common choice for any data communication because of simplicity of installation of network. The IEEE 802.11 is a simple example of the use of wireless LAN. This system is now available in the daily life of the person. At similar time the industrial plant has also used the wireless network for monitoring the various parameter and control the process. The various sensor nodes are connected to sink and sink may be connect over local PC or remote PC over internet for monitoring the experimental area. A wireless sensor network (WSN) in its simplest form can be defined as a network of (possibly low-size and less complex) devices which are denoted as nodes that can sense the environment and communicate the information gathered from the monitored field through wireless channel or link. The data is forwarded, possibly via multiple hops relaying, to a sink that can use it locally, or connected to other networks (e.g., the Internet).figure 1 shows the data communication for WSN

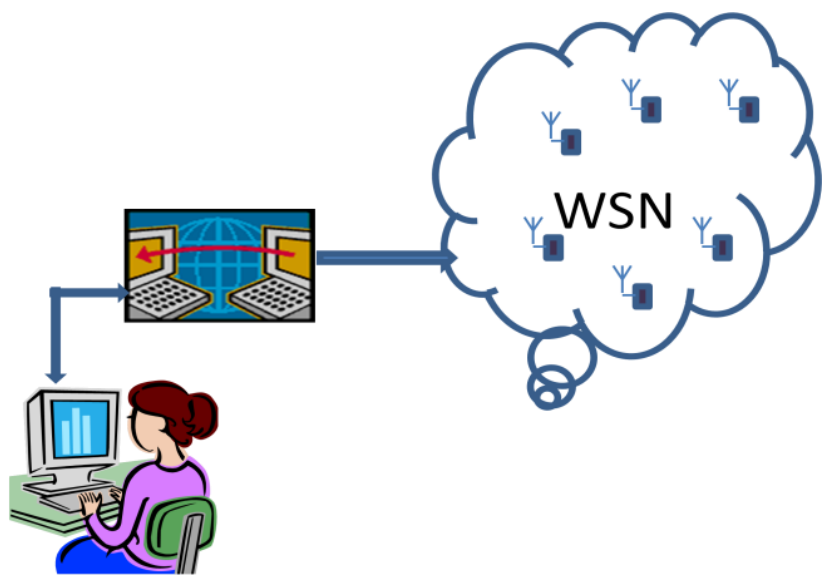

Figure 1: Data acquisition from WSN

The idea of development of wireless sensor networks was initially motivated by military applications. A WSN provides a reliable, low maintenance, low power method for making measurements in applications where cabled sensors are impractical or otherwise undesirable. The wireless sensor networks are interesting network to study due to the fact that large number of applications are being developed using these networks. A wireless sensor network of the type investigated here refers to a collection of sensors, or nodes that are linked by a medium which is wireless in nature. Connections between nodes may be formed using such media as infrared devices or radios. Wireless sensor networks will be used for such tasks as surveillance, widespread environmental sampling, security and health monitoring. They can be used in almost any environment, even those where wired connections are not possible, where the terrain is inhospitable, or where physical placement is difficult.

Wireless sensor networks are quite challenging networks as resources are limited and different network topologies is possible.

A sensor node is usually limited in terms of sensing and computation capabilities , communication performance and energy requiring a large sensor nodes for collecting information

The decrease in size and cost of sensor nodes has made it possible to have a network of large number of sensor nodes, thereby increasing the reliability and accuracy of data as well as the area of coverage. Due to the redundancy network fault tolerance increases .such redundancy also increases the network fault tolerance as the failure of single node has negligible effect on entire network application. 
One of the main critical issues in WSN is optimization of communication cost and overhead. Large efforts are being made to optimize or minimize the communications overheads. In [1] authors have demonstrated the optimization of communication overhead and its variation with respect to node velocity and network length In [2] the variation of communication overhead for BBM and cluster based techniques is evaluated. Due to dynamic nature of wireless sensor networks e.g in environment monitoring application and in military surveillance, resource optimization is very important. Establishing a secure communication link in a wireless sensor network is a challenging task due to resource limitation and wireless nature of transmission, and failure free communication establishment between source and sink is affected due to which reliability of the information is affected.

\section{RELIABILITY OF WSN}

In Wireless sensor network critical event data collected by the sensor nodes need to be reliably delivered to the sink for successful monitoring of an environment. Therefore, given the nature of error prone wireless links, ensuring reliable transfer of data from resource constrained sensor nodes to the sink is one of the major challenges in WSNs. Reliable transfer of data is the surety that the packet carrying event's information arrives at the destination.

Many protocols have been proposed to address the reliability issue in wireless sensor networks; each of them proposes deferent ways of reliably transporting data packets from sensor node to the sink. Due to the convergent nature of traffic in WSNs, all sensor nodes in the network tends to inject their captured data towards the sink.

One way of achieving reliability in terms of recovering the lost packets is through the use of retransmissions of the lost packets. Retransmissions can either be performed on end-toend or hop-by-hop basis. End-to-end re-transmission requires only the source node that generated the packet to re-transmit the lost packet. Whereas, hop-by-hop retransmission allows the intermediate nodes to perform retransmission of lost packets by caching the information

Another way to achieve data transport reliability is by introducing information redundancy where multiple copies of the same packet are transmitted based on erasures codes that allows receiver to recover from independent packet loss [3]. Iyer et al. [5] proposed Sensor Transmission Control Protocol (STCP)which is a generic (sensor-to-sink) end-to-end transport protocol providing both congestion control and reliability.

\section{WSN NODES CLUSTERING}

Grouping sensor nodes into clusters has been widely pursued by the research community in order to achieve the network scalability objective. Every cluster would have a

leader, often referred to as the access point or cluster-head. Although many clustering algorithms have been proposed in the literature for ad-hoc networks [7-10], the objective was mainly to generate stable clusters in environments with mobile nodes. Many of such techniques care mostly about node reachability and route stability, without much concern about critical design goals of WSNs such as network longevity and coverage. Recently, a number of clustering algorithms have been specifically designed for WSNs [1217]. These proposed clustering techniques widely vary depending on the node deployment and bootstrapping schemes, the pursued network architecture, the characteristics of the cluster head $(\mathrm{CH})$ nodes and the network operation model. A CH may be elected by the sensors in a cluster or pre-assigned by the network designer. A $\mathrm{CH}$ may also be just one of the sensors or a node that is richer in resources. The cluster membership may be fixed or variable. $\mathrm{CHs}$ may form a second tier network or may just ship the data to interested parties, e.g. a base-station or a command center.

The primary means of relaying data among nodes in WSN, whether clustered or non-clustered is via a routing protocol [6-12]. Hence, an essential and critical design requirement of the routing protocol is that it be energy-aware. An energyaware routing protocol should exhibit energy efficiency and balanced energy consumption across the WSN. The first requirement ensures that the WSN can sustain operations over pro-longed, unattended periods. The latter requirement ensures that sections of the WSN do not fail prematurely and disrupt operations.

Wireless sensor nodes that have variable-powered RF transceivers can provide greater routing performance at the cost of higher power consumption [12-17]. On the other hand, nodes that have fixed-power RF transceivers are generally cheaper but may be more prone to communication disruptions. Even large advances in Micro Electro Mechanical Systems (MEMS) technology, energy constraints continue to limit the operations lifetime of a WSN and new, energy-aware motes are still in research stage.

LEACH, TEEN, APTEEN [18-21] are cluster based routing protocols, whereas PEGASIS is a chain-based protocol. The performance of APTEEN lies between TEEN and LEACH with respect to energy consumption and longevity of the network [18]. TEEN only transmits time-critical data, while APTEEN performs periodic data transmissions. In this respect APTEEN is also better than LEACH because APTEEN transmits data based on a threshold value whereas LEACH transmits data continuously. Again PEGASIS avoids the formation of clustering overhead of LEACH, but it requires dynamic topology adjustment since sensor energy is not tracked. PEGASIS introduces excessive delay for distant nodes on the chain. Single leader can become a bottleneck in PEGASIS. PEGASIS increases network lifetime two-fold compared to the LEACH protocol.

The architecture of BBM is based on random propagation of information from source to sink as shown in the figure 2. The Hexagonal topology is illustrated in BBM techniques [2]. Using MATLAB the information flows from source node 5 to sink node 88 is illustrated with shortest path routing. Network length is $100 \times 100$. 


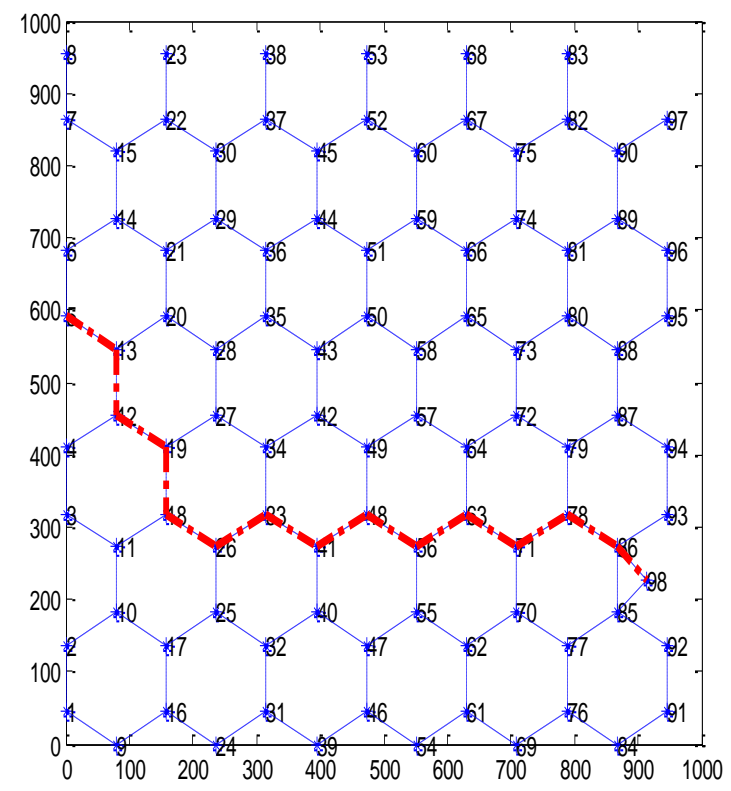

Figure 2: BBM hexagonal distribution of access points

In the following figure -3 the same 1 WSN network length 100x100 node is illustrated in clustering method with 5 clusters and source node 5 and sink node 8 .

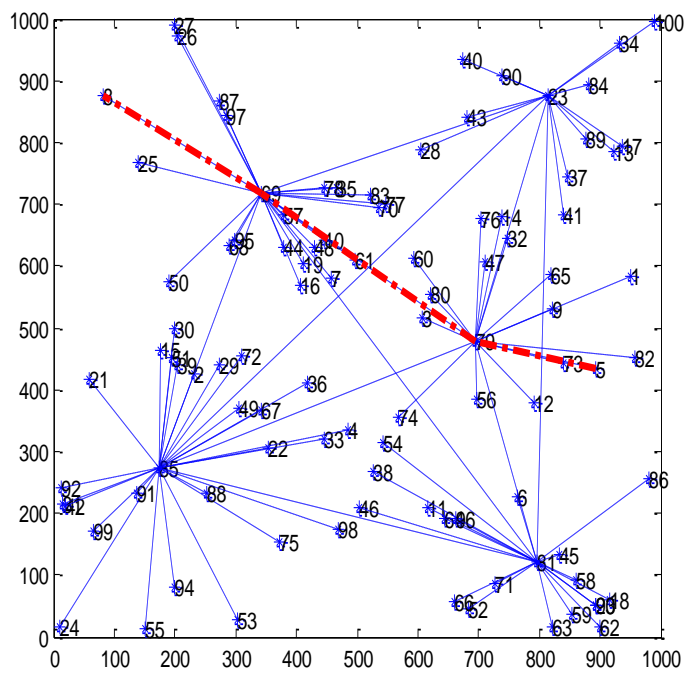

\section{Figure 3: Cluster based approach for 5 access points}

\section{Mathematical description for BBM}

Let $\mathrm{N}$ is the no of node in full LxL network area.

The Packet transmission by each node during the network establishment is: $=\mathrm{N}(\mathrm{N}-1)$

The transmission by sink node to all other node is updated in time $\mathrm{t}$ second

The overhead per second is $=(\mathrm{N}-1) / \mathrm{t}_{\mathrm{u}}$
With movement of every d meter from the original location of the sink , the extra communication is required $=(\mathrm{N}-1) / \mathrm{d}_{\mathrm{u}}$

The communication overhead with time $\mathrm{t}=\left(\mathrm{N} / \mathrm{t}_{\mathrm{u}}\right){ }^{*} \mathrm{t}$

The communication overhead with distance moved $d$ is $==\left(\mathrm{N} / \mathrm{d}_{\mathrm{u}}\right) * \mathrm{~d}$

With velocity $\mathrm{V}=\mathrm{d} / \mathrm{t}$ the communication overhead $=\left(\mathrm{N} / \mathrm{t}_{\mathrm{u}}\right) * \mathrm{t}+\left(\mathrm{N} / \mathrm{d}_{\mathrm{u}}\right) * \mathrm{~d}$

$=\left(\mathrm{N} / \mathrm{t}_{\mathrm{u}}\right) * \mathrm{t}+\left(\mathrm{N} / \mathrm{d}_{\mathrm{u}}\right) * \mathrm{~V} * \mathrm{t}$

The total communication overhead in BBM technique $=\left(\mathrm{N} / \mathrm{t}_{\mathrm{u}}\right) * \mathrm{t}+\left(\mathrm{N} / \mathrm{d}_{\mathrm{u}}\right) * \mathrm{~V} * \mathrm{t}+\mathrm{N}(\mathrm{N}-1)$.

Mathematical description for Clustering

Let $\mathrm{N}_{\mathrm{c}}$ is the no of cluster head of each cluster Having node $\mathrm{N}_{1}, \mathrm{~N}_{2}, \mathrm{~N}_{3}, \ldots \ldots . \mathrm{N}_{\mathrm{c}}$.

The Packet transmission by each node during the network establishment is:

$=\mathrm{N}_{1}\left(\mathrm{~N}_{1}-1\right)+\mathrm{N}_{1}\left(\mathrm{~N}_{1}-1\right)+\ldots \ldots \ldots \ldots . . . \mathrm{N}_{\mathrm{Nc}}\left(\mathrm{N}_{\mathrm{Nc}}-1\right)$

The transmission by sink node to all cluster head node is updated in time $\mathrm{t}$ second

The communication overhead per with time $\mathrm{t}=\left(\mathrm{N}_{\mathrm{c}} / \mathrm{t}_{\mathrm{u}}\right) * \mathrm{t}$

With movement of every $d$ meter from the original location of the sink, the extra communication is required $=\left(\mathrm{N}_{\mathrm{c}} / \mathrm{d}_{\mathrm{u}}\right) * \mathrm{~d}$

With velocity $\mathrm{V}=\mathrm{d} / \mathrm{t}$ the communication overhead $=\left(\mathrm{N}_{\mathrm{c}} / \mathrm{t}_{\mathrm{u}}\right) * \mathrm{t}+\left(\mathrm{N}_{\mathrm{c}} / \mathrm{d}_{\mathrm{u}}\right) * \mathrm{~d}$

$=\left(\mathrm{N}_{\mathrm{c}} / \mathrm{t}_{\mathrm{u}}\right) * \mathrm{t}+\left(\mathrm{N}_{\mathrm{c}} / \mathrm{d}_{\mathrm{u}}\right) * \mathrm{~V} * \mathrm{t}$

The total communication overhead in clustering technique $=\left(\mathrm{N}_{\mathrm{c}} / \mathrm{t}_{\mathrm{u}}\right) * \mathrm{t}+\left(\mathrm{N}_{\mathrm{c}} / \mathrm{d}_{\mathrm{u}}\right) * \mathrm{~d}+\mathrm{N}_{1}\left(\mathrm{~N}_{1}-1\right)+\ldots \ldots . . \mathrm{N}_{\mathrm{Nc}}\left(\mathrm{N}_{\mathrm{Nc}}-1\right)$

\section{SIMULATION RESULTS}

In comparative communication overhead evaluation shows that overhead is reduced in clustering [2]. The result is shown in figure 5 for communication overhead. Figure 3 shows the topology of access points in hexagonal distribution. Figure 4 shows the physical layout of nodes spread over a 1000 meter square area with 5 access points for clustering. Simulation was carried out in MATLAB [22].

Simulation parameters for velocity vs. communication overhead are taken as follows.

- No. of nodes: 100

- $\quad$ Sink : single

- Update time : $10 \mathrm{sec}$

- Velocity : $10 \mathrm{~m} / \mathrm{s}$ to $100 \mathrm{~m} / \mathrm{s}$ 
Communication overhead in WSN with diffrent velocity for BBM and d luster based protocol

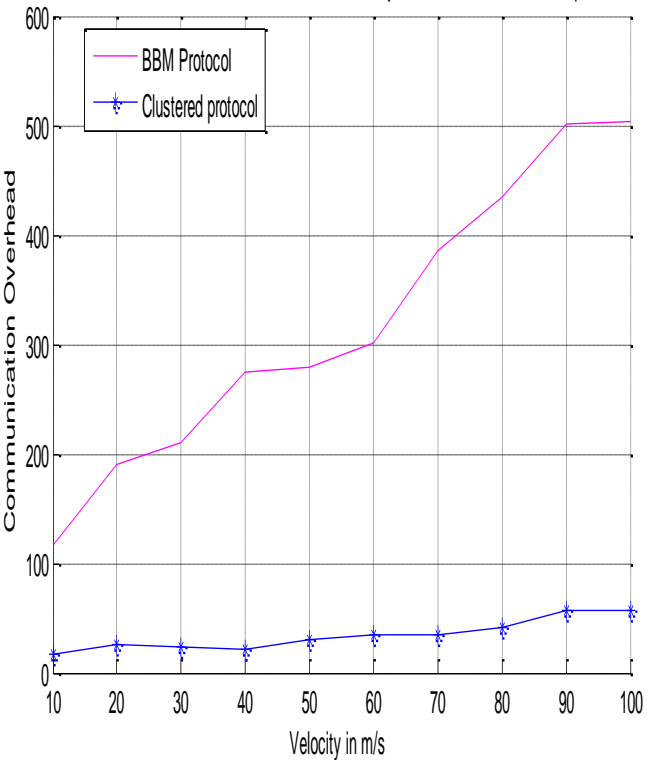

Figure 4: Communication overhead in WSN with different velocity for BBM and cluster based protocol

As can be seen from figure 4 the communication overhead in cluster based protocol is much less than the Broadcast based method ( BBM) based protocol as the velocity of nodes increases.

Figure 5 shows the result and comparison of two approaches. As can be seen from figure 6 the reliability ,the cluster approach maintains the trade off of reliability and energy consumption by optimizing the cluster number (communication distance). Relaibility of cluster based approach does not much influenced by velocity of sink node. protocol as the velocity of nodes increases. In BBM protocol overhead increases (fig-5)almost linearly with nodes velocity. These simulation results indicates that cluster based approachl outperforms the BBM based approach in the context of communication overhead with trade off of reliability with node density.

\section{CONCLUSION}

Wireless Sensor Networks are used for monitoring and collecting information from an unattended environment and for reporting events to the user. They monitor physical or environmental conditions such as temperature, humidity, pressure, sound, vibration. The reduction of energy overhead is a major challenge in wireless sensor networks. The energy overhead reduction is normally suffer with poor reliability due to energy depletion of the communicating nodes. The reliability is slightly better in case of $\mathrm{BBM}$ for $\mathrm{N}=20$ as compared with cluster technique for node $=20, \mathrm{Nc}=4$. The $\mathrm{BBM}$ reliability is of the order of 0.9 while clustering it is of the order of .8 although of not much significant decay. In clustering reliability decreases up to 0.2 after increasing the no of node more than 100 in case of BBM reliability does affected but in case of Clustering decreases. The cluster approach will maintain the trade off of reliability and energy consumption by optimizing the cluster number (communication distance). In this paper, the reliability and communication overhead has been demonstrated with the sink velocity and Node density.

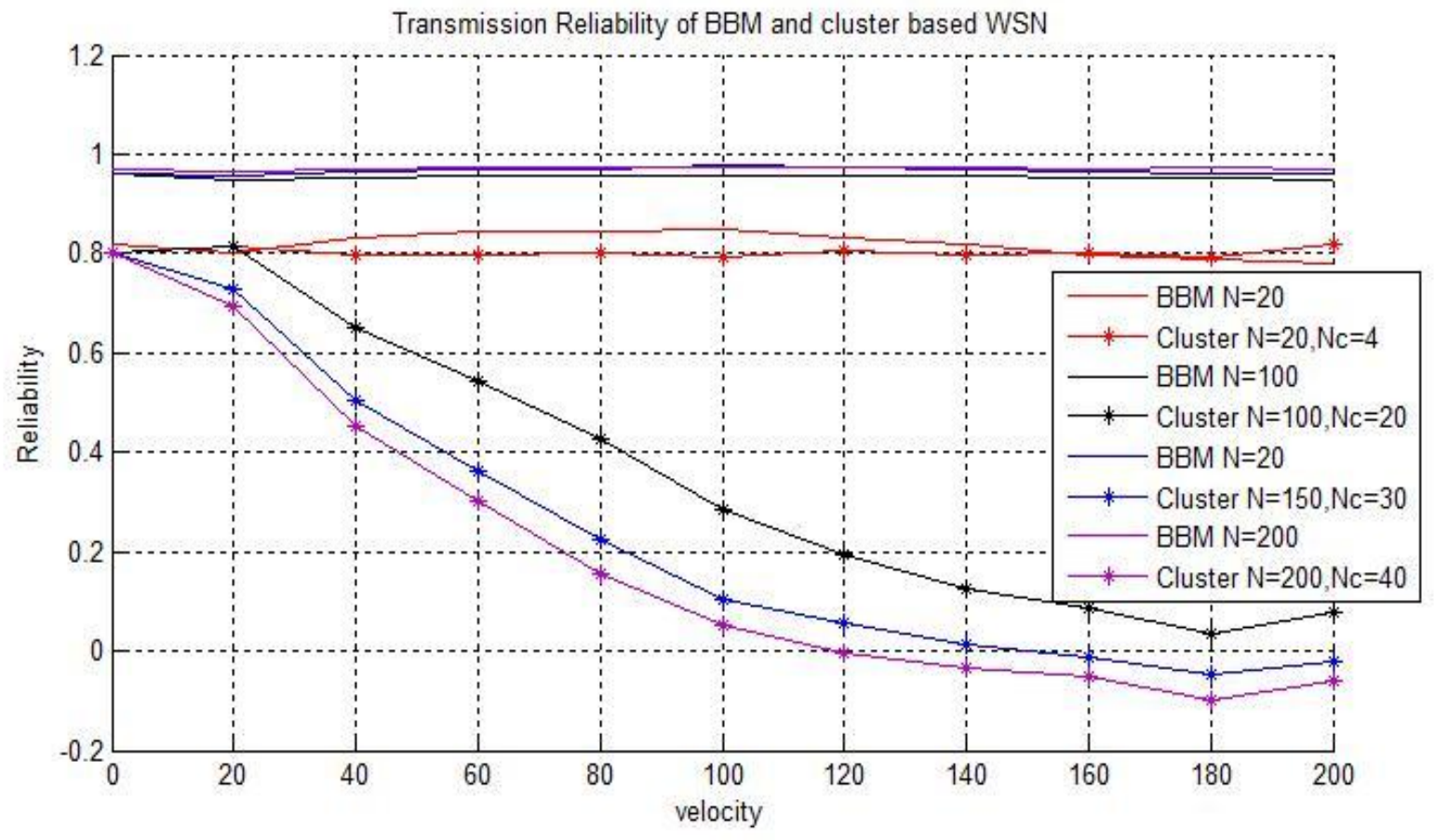

Figure 5 : Comparison of BBM and cluster based approach 


\section{ACKNOWLEDGMENTS}

Authors are thankful to the Head of institute, JMGP Burhanpur, India for his kind support and cooperation during the course of this work, Thanks are also due to friends and colleagues of our department for their cooperation, fruitful technical discussion and guidance.

\section{REFERENCES}

[1] Shiv Prasad Kori and Dr R K baghel "Evaluation of Communication Overheads in Wireless Sensor Networks "International Journal ofEngineering Research(ISSN : 23196890) Volume No.2, Issue No.2, pp :167-171 01 April 2013.

[2] Shiv Prasad Kori and $\operatorname{Dr} \mathrm{R} \quad \mathrm{K}$ baghel, "PerformanceComparison in Terms of Communication Overhead for Wireless sensor Network Based on Clustering Technique" IJECCE (ISSN : 2249-074X) Volume No.4, Issue No.3,pp : 743-746

[3] Suganthi, K.; Sundaram, B.V.; Kumar, K.S.V.; Ashim, J.S.; Kumar, S.S.; ,"Improving energy efficiency and reliability using multiple mobile sinks and hierarchical clustering in wireless sensor networks," Recent Trends in Information Technology (ICRTIT), 2011 International Conference on, vol., no., pp.257-262, 3-5 June 2011.

[4] L. Rizzo, Effective erasure codes for reliable computer communication protocols, ACM Computer communication Review, vol. 27, no. 02,pp. 24 \{ 36, 1997.

[5] Y. Iyer, S. Gandham and S. Venkatesan, STCP: a generic transportlayer protocol for wireless sensor networks," in Proceedings of 14th International Conference on Computer Communications and Networks (IC-CCN), San Diego, California, USA, pp. 449 \{ 454, 17-19 October2005.

[6] S. Bandyopadhyay and E. J. Coyle, "An energy efficient hierarchical clustering algorithm for wireless sensor networks,” IEEE Infocom '03, pp. 1713-1723, 2003.

[7] Q. Li, J. Aslam, and D. Rus, "Online power-aware routing in wireless ad hoc networks," IEEE/ACM International Conference on Mobile Computing and Networking (MobiCom 2001), Rome, Italy, pp. 97-107, July 2001.

[8] J. H. Chang and L. Tassiulas, "Energy conserving routing in wireless ad-hoc networks," INFOCOM, pp. 22-31, 2000.

[9] T. H. Lin, Y. S. Chen, and S. L. Lee, "PCAR: A power aware chessboard-based adaptive routing protocol for wireless sensor networks," IEEE 6th CAS Symposium on Emerging Technologies, pp. 145-148, 2004.

[10] M. Perillo and W. Heinzelman, "Dapr: A protocol for wireless sensor networks utilizing an application-based routing cost," IEEE Wireless Communications and Networking Conference (WCNC), pp. 1540-1545, 2004.

[11] M. A Youssef, M. F. Younis, and K. A. Arisha, "A constrained shortest-path energy-aware routing algorithm for wireless sensor networks," WCNC 2002-IEEE Wireless Communications and Networking Conference, No. 1, pp. 682-687, March 2002

[12] A. Manjeshwar and D. Agrawal, "TEEN: A routing protocol for enhanced efficiency in wireless sensor net-works," in Proceedings of the 15th International Parallel \& Distributed Processing Symposium.

[13] http://www.ctr.kcl.ac.uk/iwwan2005/papers/57_not_attende d.pdf.
[14] A. Manjeshwar and D. P. Agrawal, "APTEEN: A hybrid protocol for efficient routing and comprehensive information retrieval in wireless sensor networks," Proceedings of International Parallel and Distributed Processing Symposium (IPDPS'02), pp. 195-202, 2002.

[15] J. Kamimura, N. Wakamiya, and M. Murata, "Energyefficient clustering method for data gathering in sensor networks," Proceedings of First Annual International Conference on Broadband Networks 2004, pp. 1-10, 2004.

[16] R. C. Shah and J. M. Rabaey, "Energy aware routing for low energy adhoc sensor networks," Proceedings of IEEE Wireless Communications and Network Conference, Vol. 1, pp. 350-355, March 2002.

[17] J. Chen, Y. Guan, and U. Pooch, "Customizing a geographical routing protocol for wireless sensor networks," Proceedings of International Conference on IT: Coding and Computing (ITCC,'05), pp. 586-591, 2005

[18] Jamal N. Al-Karaki, "Routing Techniques in Wireless Sensor Networks: A Survey", IEEE Wireless Communications, Vol. 2, No. 2, December 2004, pp.704709 .

[19] Raju Pal, Ajay K. Sharma, "MSEP-E: Enhanced Stable Election Protocol with Multihop Communication", IEEE Conference on Asia-Pacific Service Computing Conference, Vol. 2, Issue. 2, February 2011, pp 172-178.

[20] K.Suganthi, Dr.B.VinayagaSundaram, K.S.Vishwa Kumar, J.SyedAshim, S.Saravana Kumar, "Improving Energy Efficiency and Reliability using multiple mobile sinks and hierarchical clustering in Wireless Sensor Networks", IEEEInternational Conference on Recent Trends in Information Technology, MIT, Anna University, Chennai, Vol.5, No.4, June 3-5, 2011.

[21] Z. Maria Wang, Stefano Basagni, Emanuel Melachrinoudis and Chiara Petriol, "Exploiting Sink Mobility for Maximizing Sensor Networks Lifetime" IEEE Proceedings of the 38th Hawaii International Conference on System Sciences, pp. 1-5, March 2005.

[22] FahedAwad, EyadTaqieddin, AsmaaSeyam," EnergyEfficient and Coverage-Aware Clustering in Wireless Sensor Networks", Wireless Engineering and Technology, SciRes, Vol. 3, 2012, pp.142

[23] Mathsworks(http://www.mathworks.com)

\section{Authors Profile}

Shri Shiv Prasad Kori received the B.E. degree in electronics and telecommunication Engineering in 1994 from G.E.C. Jabalpur. India and $M$. Tech degree in Digital systems from MNNIT Allahabad in 2003. He is working as senior faculty in JM Polytechnic Burhanpur(M. P) and pursuing his Ph.D from MANIT Bhopal.

Dr. R.K Baghel received the B.E degree in Electronics in 1990 the M.TECH degree in Digital signal Processing in 1997, and Ph.D in Optical communication from University of Bhopal. From 1992-2000 he involved in the equipment development for syncronus optical development. Recently he is involved in low pressure measurement equipment using capacitive transducer and LCD/LED driver structure development for low power high speed \& their power management. He is Professor in MANIT BHOPAL from 1991-till date. He has authored or coauthored over 15 refereed international journal and conference papers. 